\title{
Das chirurgische Schattenkabinett
}

\section{Dominik Heim}

PD Dr. med., Suva, Luzern

Im November 2014 fand im Kantonsspital Olten die 40-Jahr-Feier des Oltener Chirurgen Clubs OCC statt. Was bzw. wer ist der OCC?

\section{Die Gründung}

1974 schrieb Walter Laube, Chefarzt der Chirurgie am Kantonsspital Olten, «Schon seit mehreren Jahren zeigte sich unter den chirurgischen Chefärzten von kleineren und mittleren Akutspitälern das Bedürfnis zu einem engeren Kontakt untereinander.» Und so trafen sich am 22. Juni 1974 in Olten vierzehn Chirurgen. Man wolle «keinen Extrazug in Konkurrenz zu einer bestehenden Organisation bilden» wurde betont. Otto Wicki, Gründungsmitglied und Chefarzt am Spital Wolhusen, schrieb zwar an Franz Deucher, den damaligen Präsidenten der Schweizerischen Gesellschaft für Chirurgie, SGC, «Ich bin der Auffassung, dass in der neugeplanten Ordnung der FMH-Ausbildung die Universitätsspitäler ein allzu grosses Gewicht bekommen». Kämpferisch betonte er an der Gründungssitzung «Die Chefärzte der Regionalspitäler haben nichts zu sagen in der Chirurgengesellschaft». Das Protokoll schwächt dann ab: «Die Gruppe soll vor allem ein fachliches Diskussionsplateau sein und auch Gelegenheit geben zur Behandlung von Standesfragen»

\section{Der Zweck}

Als Hauptpunkt sei aber ursprünglich nicht die Wissenschaft geplant gewesen, sondern die Standespolitik und die gegenseitige standespolitische Hilfe, sagt Wicki heute. Die medizinischen Vorträge hätten in der Folge als Zugpferd für eine grössere Teilnahme gedient. Noch zügiger sei das Versprechen von Kaffee und Gipfeli nebst Aperitiv und feinem Gratis-Mittagessen mit gemütlichem Zusammensein gewesen! Die zweite Tagung in Wolhusen zeigte dann in der Themenwahl das fachliche, breite Spektrum des OCC: «Pylorotomie beim Erwachsenen», «Pancreasruptur», «Thoraxverletzung und Intensivpflege», aber auch «Symphysenruptur» und von August Guggenbühl, einem frühen Mitglied der AO, «Bemerkungen zur Plattenosteosynthese». Einladungen gingen auch an den Urologen (und Chirurgen) Hubert Mäder am Bürgerspital Zug und an den Orthopäden Andreas Burkhardt, denn für «solche Fragen hätten wir gerne bei der Diskussion einen Fachkollegen aus der Orthopädie gehört». Später wurde dann auch Hans Zollinger, Chefarzt der Suva, eingeladen. Der OCC suchte den interdisziplinären Dialog.

\section{Der Club}

Zwei Tagungen gab es jährlich. Die Geschäftssitzung wurde während des Mittagessens durchgeführt. Einen Mitgliederbeitrag gab es nicht. Wer mehr als zwei Mal unentschuldigt fehlte, erhielt keine Einladung mehr. Einen Namen hatte dieser Verein «der kein Verein sein soll» (Laube) anfänglich nicht, man einigte sich 1975 an der Tagung in Menziken auf "Oltener Chirurgen Club", OCC. 1983 zählte der OCC 53 Mitglieder, heute sind es 137. In erster Linie waren es Chefärzte und Co-Chefärzte aus der Deutschschweiz, aber mit St. Croix, Lugano und Acquarossa waren auch nicht deutschsprachige Regionen im OCC vertreten. Einen Vorstand hatte der OCC nicht, die Akten und die Ordner waren beim jeweiligen Tagungspräsidenten. 1986 dann der Schreck, «oh weh,

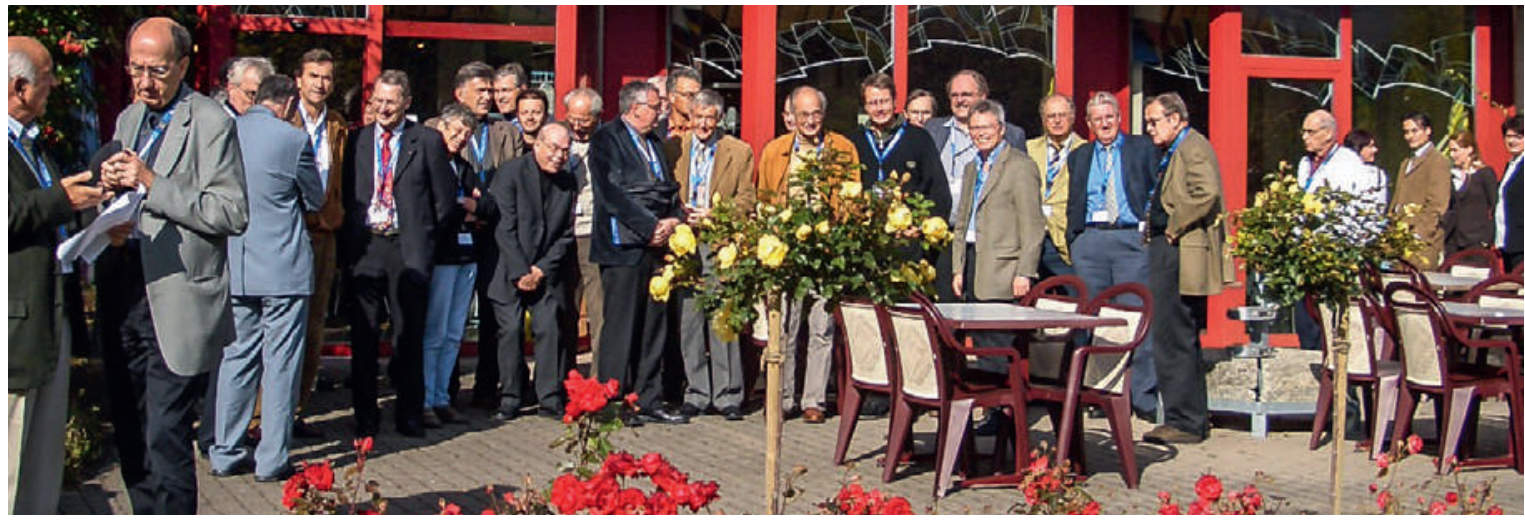

Der Oltener Chirurgen Club im Jahr 2007 in Frutigen. 
verloren ging das Club-Buch». Zwei Jahre später tauchte es mirakulös wieder auf! Auch Statuten hatte der OCC nicht. Es gab die 10 ungeschriebenen Regeln und die zehnte hiess «wir sagen uns alle Du». Im Protokoll 1986 von Aarburg wurde dazu vermerkt: «Das «per Du〉 ist kein Thema, sondern eine Selbstverständlichkeit!» Auf dieser ganz einfachen «Vereinsstruktur» basierte diese Vereinigung von Allgemeinchirurgen aus kleineren und mittelgrossen Spitälern. Nicht zum ersten Mal in der Schweiz entstand ganz Entscheidendes im Gesundheitswesen in der Peripherie [1, 2]. Und diese Impulse wurden geschätzt «... die Alternative ist die unmögliche Forderung nach zahlreichen «unterbeschäftigten〉 engsten Spezialisten. Umso wichtiger ist es, dass ausserhalb grosser Zentren an all jenen Kliniken, wo auch eine breite chirurgische Versorgung betrieben werden kann, eine weitere Aufteilung in chirurgische und orthopädischen Chefärzte abgeblockt werden kann", schrieb Felix Harder, Präsident der SGC, 1989. Folgerichtig war dann das Tagungsthema in Grosshöchstetten im Frühling 1991 «Das Berufsbild des Allgemeinchirurgen heute und morgen" und im Herbst in Samedan «Die Grauzone Traumatologie der Extremitäten».

\section{Die Auswirkungen}

Das Thema «Traumatologie in der Allgemeinchirurgie» blieb aktuell. Man nannte den OCC in dieser Zeit nicht ganz zu Unrecht ein «unruhestiftendes Schattenkabinett» (Biaggi): Im April 1995 wurde in Rheinfelden die gefährliche Tendenz «zur Abwertung des Allgemeinchirurgen» diskutiert. Im Oktober wurde in Brig über die eventuelle Gründung einer Schweizerischen Gesellschaft für Allgemeinchirurgie diskutiert. Und am 24. Mai 1996 wurde dann in Baden im Zuge der Erweiterung des Facharzttitels Chirurgie die Schweizerische Gesellschaft für Allgemeine Chirurgie, SGAC, gegründet. Ihr erster Präsident, Gabriel Ayer, Chefarzt in Wil, erklärte den SGC-Mitgliedern die Notwendigkeit dieser neuen Gesellschaft mit dem Zitat von J. R. Siewert, München: «Niemand wollte die allgemeine Chirurgie - jetzt, da sie verschwunden ist, herrscht Ratlosigkeit». Aber Ratlosigkeit wollte man nicht! Der OCC blieb: Er verfügte jetzt über eine verstärkte Position der Allgemeinchirurgie im SGC Vorstand. Er formulierte im Unionsbulletin die Probleme der Allgemeinchirurgie in der Rubrik «die Seite des B-Spitals». Und er trug wesentlich zur erwähnten Gründung der SGAC, heute SGACT, Schweizerische Gesellschaft für Allgemeinchirurgie und Traumatologie, bei. Im Januar 2003 wurde der Schwerpunkttitel «Allgemein- und Unfallchirurgie» als einer der fünf Schwerpunkttitel (Viszeralchirurgie, Gefässchirurgie, Thoraxchirurgie und Handchirurgie) von der Schweizerischen Ärztekammer auf der Basis der neuen Weiterbildungsordnung der FMH anerkannt.

\section{Die Jahre danach}

Brisante Themen beschäftigten den OCC auch in den folgenden Jahren: Frühzeitiges Ausscheiden von Chef- und Co-Chefärzten an Spitälern (Tagung in Affoltern), Burnout (Tagung in Münsingen) und Tagesklinik (Tagung in Oberdiessbach). «Man war bestürzt über das knallharte Kostenmanagement. Die Zahlen allein entscheiden. Das Wohl des Patienten und die ärztliche Ethik sind nicht mehr gefragt», wurde moniert. Der Umgang mit dem ärztlichen Kader wurde kritisiert «kein effizient geführter Betrieb kann es sich leisten, seine Mitarbeiterinnen und Mitarbeiter in einer Art und Weise zu demotivieren, wie es heute mit den Ärzten und Ärztinnen geschieht» (R. Bärfuss, Direktor EGK, Herbsttagung 2000 in Sumiswald).

\section{Wie gehts weiter?}

"Ich denke, dass der OCC, wenn er lebendig bleiben will, wieder vermehrt Akzente auf die politischen, standespolitischen und organisatorischen Probleme von Spitalärzten setzen sollte» (Jean Biaggi). Eine sehr berechtigte Idee angesichts der sinkenden Wertschätzung der ärztlichen Kaderarbeit durch die Spitalführungen. «Mit der Gründung des Schwerpunktes für Allgemeinchirurgie und Traumatologie ist vorab der wissenschaftliche Aspekt abgedeckt", auch das tönt mit Blick auf die Bieler Fortbildungstage berechtigt. Und da war aber noch mehr: «Alljährlich gibt es eine Herbsttagung. Die Tagungsorte wechseln jedes Mal, damit wir die Spitäler der Mitglieder kennenlernen" (Punkt 6 der ungeschriebenen Regeln) der freundschaftliche Kontakt in der Schicksalsgemeinschaft "Allgemeinchirurgie»! In der zerklüfteten Schweizer Spitallandschaft hat die "Chirurgie des Häufigen" nach wie vor einen hohen Stellenwert, deshalb braucht es auch heute diesen Oltener Chirurgen Club. Nicht aus Nostalgie, sondern als Zusammenhalt der Allgemeinchirurgen an kleineren und mittleren Akutspitälern, so wie es Walter Laube vor 40 Jahren formuliert hat.

\section{Danksagung}

Ich danke Dr. Jean Biaggi, Dr. Otto Wicki und Dr. Wolf Zimmerli für die Durchsicht des Manuskripts.

\section{Referenzen}

1 Heim D. In memoriam Reinhard Fischer. Schweiz Ärztezeitung. 2013:94(4):97.

2 Heim D. SGTV - eine interdisziplinäre Gesellschaft mit europäischer Vernetzung. Schweiz Ärztezeitung. 2008;89(16):696-7.

Weitere Quellen

- OCC-Ordner von Dr. Otto Wicki, Iragna.

- «Wandernde» OCC-Ordner im Besitz der jeweiligen Tagungspräsidenten. 